\title{
Iniquidades na assistência odontológica ao longo do curso de vida de jovens: um estudo de coorte
}

\author{
Inequalities in dental care during the life course of young people: \\ a cohort study
}

Ana Karine Macedo Teixeira ${ }^{1}$

Angelo Giuseppe Roncalli ${ }^{2}$

Luiz Roberto Augusto Noro ${ }^{2}$

${ }^{1}$ Departamento de Clínica Odontológica, Universidade Federal do Ceará. R.

Monsenhor Furtado s/n, Rodolfo Teófilo. 600430355 Fortaleza CE Brasil. anakarinemt@hotmail.com ${ }^{2}$ Departamento de Odontologia, Universidade Federal do Rio Grande do Norte. Natal RN Brasil.
Abstract The scope of this study was to investigate dental care from the life course perspective and its determinant factors among young people in the city of Sobral, State of Ceará, Brazil. A cohort study was conducted with waves in 2000, 2006 and 2012 with 482 young people aged between 17 and 21 years. Two outcomes were investigated: immediate dental care and the lack of dental care, based on the dental trajectory in relation to restorative treatment. Socioeconomic conditions and recourse to oral health services and actions in the three waves investigated were used as independent variables. It was found that low socioeconomic status throughout life presented itself as a risk factor for lack of dental care and the inverse effect was observed with immediate dental care. Participation in groups of teenagers was also related to dental care, as well as receiving information on oral health. This study revealed the presence of inequalities in dental care throughout the life course of the population assessed.

Key words Dental care, Health inequalities, Oral health

Resumo O objetivo deste estudo foi investigar a assistência odontológica sob a perspectiva do "life course", bem como seus fatores determinantes, em jovens no município de Sobral, Ceará, Brasil. Realizou-se um estudo de coorte com ondas em 2000, 2006 e $2012 \mathrm{com} 482$ jovens atualmente entre 17 e 21 anos. Foram investigados dois desfechos: a assistência odontológica imediata e a ausência de assistência odontológica, baseados na trajetória dentária em relação ao tratamento restaurador. E como variáveis independentes, utilizaram-se as condições socioeconômicas e utilização de serviços e ações de saúde bucal nas três ondas investigadas. Observou-se que piores condições socioeconômicas ao longo da vida apresentaram-se como fator de risco para ausência da assistência odontológica e o efeito inverso foi observado com a imediata. A participação em grupos de adolescentes também apresentou relação com a maior assistência odontológica, bem como receber informação sobre saúde bucal. Este estudo revelou a presença de iniquidades na assistência odontológica ao longo da vida da população estudada.

Palavras-chave Assistência odontológica, Desigualdades em saúde, Saúde bucal 


\section{Introdução}

A atual política nacional de saúde bucal - Brasil Sorridente - visa reorientar o modelo de atenção em saúde bucal do país. Pautada nos princípios doutrinários do sistema de saúde brasileiro, Sistema Único de Saúde (SUS), tais como a universalidade, a equidade e a integralidade, tem como pressuposto incorporar a Saúde da Família como estratégia de reorganização da atenção básica e melhoria da qualidade de vida da população brasileira. Desde 2004, vem ampliando o acesso às ações e serviços de saúde bucal em todos os níveis de atenção à saúde, promovendo ações de promoção, prevenção, tratamento e reabilitação ${ }^{1}$.

Apesar do aumento do acesso aos serviços e ações de saúde bucal nos últimos dez anos, ainda há uma parcela da população brasileira que nunca foi ao dentista. O último levantamento epidemiológico de base nacional identificou que $18 \%$ (aproximadamente 600.000) das crianças de 12 anos e 13\% (aproximadamente 2.200.000) dos adolescentes de 15 a 19 anos nunca foram ao dentista ${ }^{2}$. Além disso, alguns autores afirmam que ainda se observam poucas mudanças no modelo assistencial em saúde bucal, apesar da inserção do dentista na Estratégia Saúde da Família e da expansão da nova política, principalmente no que se refere à incorporação de novas práticas assistenciais, integralidade do cuidado, referenciamento para outros níveis de atenção, trabalho em equipe e uso da epidemiologia para o planejamento local ${ }^{3,4}$.

A utilização dos serviços de saúde, incluindo o odontológico, é um fator determinante da saúde ${ }^{5}$, entretanto, estudos têm revelado a existência de marcantes iniquidades na utilização e acesso de serviços odontológicos no Brasi ${ }^{6,7}$. Dessa forma, faz-se necessário compreender que fatores estão relacionados com a assistência odontológica ao longo da vida do indivíduo, mesmo em sistemas considerados universais como no Brasil, visto a existência de uma parcela da população que ainda não tem acesso à assistência odontológica.

Considerando a teoria do life course (ciclo vital ou curso de vida), no qual a saúde do indivíduo é resultante de experiências prévias e atuais ao longo da vida, ou seja, uma pior situação de saúde bucal é resultante da interação de múltiplos fatores, desde privação socioeconômica a problemas de saúde, ao longo do curso de vida do indivíduo, o que reforça a necessidade de estudos longitudinais ${ }^{8,9}$.

No município de Sobral (CE), localizado no Nordeste brasileiro, foi realizado, durante o ano
2000, um estudo transversal com 1021 crianças de cinco a nove anos. Constatou-se ainda que $23 \%$ das crianças aos cinco anos apresentaram-se livres de cárie e que 50\% dessa população tiveram acesso ao serviço dentário pelo menos uma vez na vida e que a maioria utilizou o serviço público $^{10,11}$. Em 2006, dando seguimento ao primeiro estudo no mesmo município, um levantamento epidemiológico da incidência da cárie dentária foi realizado com os adolescentes de 11 a 15 anos de idade, os mesmos que participaram da pesquisa em 2000, no qual se verificou uma incidência média de 1,86 dentes cariados por adolescente ${ }^{12}$. Em 2012, deu-se seguimento à terceira onda da coorte de saúde bucal do município de Sobral.

Portanto, o objetivo deste estudo foi investigar os fatores determinantes da assistência odontológica, sob a perspectiva do life course, de jovens no município de Sobral, Ceará, Brasil.

\section{Metodologia}

Trata-se de um estudo de coorte, desenvolvido no município de Sobral (CE), Brasil, iniciado em 2000 e com ondas nos anos de 2006 e 2012. O referencial inicial para o desenvolvimento deste estudo foi o levantamento epidemiológico de saúde bucal com a participação de 1021 crianças de cinco a nove anos, no qual foram analisadas cárie dentária, condição gengival e má oclusão. Essa amostra foi calculada considerando-se uma prevalência de má oclusão de 30\%, margem de erro de $10 \%$ e nível de confiança de $95 \%{ }^{10}$.

Seis anos depois, foi realizado um novo estudo epidemiológico em saúde bucal com caráter longitudinal, no qual se avaliou a incidência de cárie e fatores associados. Foram reexaminados 688 indivíduos, já na faixa etária de 11 a 15 $\operatorname{anos}^{12}$. Em 2012, realizou-se a terceira onda desse estudo, no qual foram investigadas as seguintes condições: incidência de cárie (índice CPOD - média de dentes cariados, perdidos e restaurados), má oclusão (Índice de Estética Dental - DAI), doença periodontal (Índice Periodontal Comunitário - IPC), traumatismo dentário e edentulismo (uso e necessidade de prótese) com os jovens na faixa etária de 17 a 21 anos.

Os indivíduos participantes da pesquisa foram identificados a partir dos agentes comunitários de saúde, que agendaram um dia para que eles comparecessem à unidade básica de saúde mais próxima da sua casa para realizar a pesquisa. Para aqueles que não compareceram no dia marcado, a equipe de pesquisa realizou até três 
visitas domiciliares. Como estratégia de busca para os que mudaram de endereço, realizaramse visitas a escolas e fábricas, além de uma busca ativa através das redes sociais.

A coleta dos dados foi realizada por sete equipes de estudantes do último ano do curso de Odontologia da Universidade Federal do Ceará Campus Sobral, sob supervisão docente, devidamente capacitados. Foi realizada a calibração interexaminadores pela técnica de consenso, com o índice Kappa variando de 0,80 a 0,93. Os exames ocorreram sob luz natural, com uso de espelho e sonda periodontal da $\mathrm{OMS}^{13}$.

Foram criados dois desfechos que expressam a trajetória da assistência odontológica ao longo do curso de vida do indivíduo, baseados na trajetória dentária em relação ao tratamento restaurador. O primeiro desfecho foi denominado assistência odontológica imediata: aplicado naquela condição em que o dente foi diagnosticado como restaurado em alguma das ondas e permaneceu restaurado em 2012. Pressupõe-se que esta condição expressa uma assistência odontológica rápida, visto que em nenhuma das ondas o dente foi diagnosticado como cariado. Portanto, foram agrupadas nesse desfecho as seguintes trajetórias dentárias ao longo da coorte (2000-2006-2012): hígido-hígido-restaurado, hígido-restaurado-restaurado e restaurado-restaurado-restaurado.

O segundo desfecho foi denominado sem assistência odontológica e indica a condição em que o dente foi diagnosticado como cariado em 2000 ou 2006 e permaneceu assim em 2012, enquadram-se nesse desfecho as trajetórias dentárias (2000-2006-2012): hígido-cariado-cariado e cariado-cariado-cariado. Portanto, este dente encontra-se cariado por seis ou mais anos sem nenhuma assistência odontológica. Esses desfechos foram definidos com o objetivo de compreender por que alguns indivíduos têm acesso imediato ao serviço odontológico e outros não.

As variáveis independentes utilizadas na análise compreenderam dados das três ondas. $\mathrm{Na}$ primeira onda (2000), durante a infância, consideraram-se as características socioeconômicas: renda familiar (até/maior que um salário mínimo), renda do chefe da família (até/maior que um salário mínimo), escolaridade do chefe da família (abaixo/acima 8 anos), tipo de construção (taipa/ alvenaria), banheiro em casa ( $\operatorname{sim} /$ não), estrutura unifamiliar (apresenta uma ou mais famílias residindo o mesmo domicílio) e trabalho infantil ( $\operatorname{sim} /$ não), utilização de serviços odontológicos: acesso ao dentista pelo menos uma vez na vida ( $\operatorname{sim} /$ não), tempo (último ano ou mais) e local da última consulta odontológica (público/privado), acesso negado ( $\operatorname{sim} /$ não) e características individuais: desnutrição infantil ( $\operatorname{sim} /$ não) e consumo de merenda escolar ( $\operatorname{sim} /$ não). Da segunda onda (2006), na adolescência, utilizaram-se as características socioeconômicas renda familiar (até/ maior que um salário mínimo), aglomeração domiciliar (até 1/mais), estudante ( $\operatorname{sim} /$ não) e tipo de escola (pública/privada) e utilização de serviços e ações de saúde bucal: acesso ao dentista pelo menos uma vez na vida, tempo (último ano ou mais), local (público/privado) e motivo (manutenção/dor ou tratamento) da última consulta, se recebeu informações de saúde bucal ( $\operatorname{sim} /$ não), acesso negado ( $\operatorname{sim} /$ não), participação de ações coletivas em saúde bucal ( $\operatorname{sim} /$ não) e se recebeu escova de dente na escola ( $\operatorname{sim} /$ não). E da terceira onda (2012), considerou-se as características sociodemográficas: sexo, renda familiar (até/maior que um salário mínimo), aglomeração domiciliar (até $1 /$ mais), estudante ( $\operatorname{sim} /$ não), tipo de escola (pública/privada), trabalho (sim/desempregado) e utilização de serviços e ações de saúde bucal: acesso ao dentista pelo menos uma vez na vida ( $\operatorname{sim} /$ não), tempo (último ano ou mais), local (público/privado) e motivo da última consulta (manutenção/dor ou tratamento) e participação em grupos de adolescentes (participação ou não em grupos formais ou informais de adolescentes, sejam de esporte, movimento cultural, religiosos ou ligados à unidade de saúde).

Para a análise dos dados, realizou-se o teste $t$ para comparação das médias dos desfechos segundo as variáveis independentes das três ondas. Calculou-se, ainda, o RR e seu intervalo de confiança para cada uma das variáveis. Por fim, realizou-se regressão de Poisson com as variáveis que apresentaram valor de $\mathrm{p}<0,20$. No modelo final somente foram mantidas as variáveis independentes que, após a regressão, apresentarem-se com nível de significância de 5\%.

Este estudo foi aprovado pelo comitê de ética da Universidade Estadual Vale do Acaraú em Sobral e os participantes assinaram termo de consentimento livre e esclarecido assegurando sua privacidade.

\section{Resultados}

No terceiro seguimento da coorte foram reexaminados 482 jovens, com taxa de resposta de $70 \%$ em relação à população encontrada em 2006. As perdas foram decorrentes de mudança de endereço (159), indivíduo não encontrado em 
casa após três visitas (24), recusa em participar (10), preso (8), morte (3) e morador de rua (1). Entretanto, essa perda não foi considerada como limitador do estudo, pois para a análise utilizouse a amostra de 482 indivíduos nos três pontos da coorte, o qual apresentou um poder de detectar um RR de até 1,2 com uma prevalência do desfecho de $60 \%$ entre os indivíduos não expostos. Tomou-se como referência valor de $\alpha=95 \%$ e poder (1- $\beta$ ) de $80 \%$.

Com relação às variáveis de utilização de serviços odontológicos, verificou-se que uma pequena parcela dos participantes, $7,5 \%$, nunca foi ao dentista e $24,7 \%$ dos jovens já tiveram acesso ao serviço odontológico negado pelo menos uma vez na vida. Em relação ao tempo da última consulta odontológica, 50,2\% visitaram o dentista há menos de um ano, em sua maioria no serviço público. Verificou-se, ainda, que 47,6\% relatou que o motivo da última consulta odontológica foi por rotina, reparo ou manutenção, entretanto $73 \%$ afirmaram já ter sentido dor de dente alguma vez na vida.

A Tabela 1 descreve os fatores relacionados com a assistência odontológica imediata, expressa pela média de dentes diagnosticados como restaurados ao longo da coorte e que permaneceram assim. Dentre as variáveis investigadas na primeira onda, constatou-se que uma maior renda familiar, maior renda e maior escolaridade do chefe da família, morar em casa de alvenaria, já ter tido acesso odontológico pelo menos uma vez na vida e se consultar no serviço privado na infância foram fatores que influenciaram o acesso odontológico imediato. Já na adolescência, uma maior renda familiar, estudar em escola privada, menor aglomeração domiciliar, ter acesso ao dentista pelo menos uma vez na vida, ter se consultado há menos de um ano e realizado consulta no serviço privado favoreceram o acesso odontológico imediato. E na juventude, as variáveis relacionadas com o acesso imediato foram: maior renda familiar, estar trabalhando, menor aglomeração domiciliar, estudar em escola privada, ter se consultado com dentista no último ano e em serviço privado, bem como participar de grupos de adolescentes.

No modelo ajustado para os fatores determinantes da assistência odontológica imediata (Tabela 2), constatou-se que maior renda do chefe da família $[R R=1,33$ (IC95\% 1,08-1,51) na infância e ter consultado dentista no último ano $[\mathrm{RR}=1,31(\mathrm{IC} 95 \%$ 1,09-1,48) $]$ determinaram uma maior assistência odontológica. Estudar em escola privada $[\mathrm{RR}=1,33(\mathrm{IC} 95 \% 1,01-1,54)]$ e ter ido ao dentista no último ano $[\mathrm{RR}=1,43$ (IC95 1,24-1,58)] na adolescência determinam um maior número de dentes com assistência na juventude. Essas variáveis foram ajustadas pela renda familiar atual. Já no modelo para as variáveis da juventude, permaneceram no modelo a maior renda familiar, menor aglomeração domiciliar, consultar o dentista no último ano e participar de grupo de adolescentes.

Com relação ao desfecho sem assistência odontológica, a Tabela 3 apresenta a sua relação com as variáveis das três ondas. Encontrou-se associação estatística com as seguintes variáveis: renda familiar, renda do chefe da família, local de consulta odontológica e desnutrição infantil na onda de 2000; aglomerado domiciliar, tipo de escola, local de consulta odontológica e se recebeu informação de saúde bucal na onda de 2006; e desemprego, aglomeração domiciliar, consulta odontológica no último ano, local da última consulta odontológica e participação em grupo de adolescente referente à onda de 2012.

Após a regressão, apenas a desnutrição infantil permaneceu no modelo para a história de dentes sem assistência odontológica, independente de o indivíduo estar desempregado atualmente. Já com relação à adolescência, estudar em escola pública apresentou 4,08 (IC95\% 1,59-10,44) vezes mais risco de não ter assistência odontológica ao longo da vida, enquanto não receber informação de saúde bucal indicou um risco de 1,71 (IC95\% 1,14-2,58) vezes, mesmo quando ajustado pelo fator desemprego atual. Nas variáveis de 2012, constatou-se que estar desempregado [RR = 1,87 (IC95\% 1,26-2,77)], aglomeração domiciliar maior que um $[\mathrm{RR}=1,61$ (IC95\% 1,09$2,39)]$, realizar consulta odontológica há mais de um ano $[\mathrm{RR}=2,47$ (IC95\%1,71-3,58)] e não ter participado de grupos de adolescentes $[\mathrm{RR}=$ $1,58(1,09-2,29)]$ permaneceram como fatores de risco para um maior número de dentes sem assistência odontológica (Tabela 4).

\section{Discussão}

Esses resultados revelaram a presença de iniquidades na assistência odontológica, visto que os jovens com as melhores condições socioeconômicas (melhor renda do chefe da família, escola privada, família com menor aglomeração domiciliar) ao longo da vida apresentaram maior possibilidade de receber assistência odontológica imediata, enquanto os que apresentaram as piores (escola pública, desemprego, maior aglome- 
Tabela 1. Média de dentes com assistência odontológica imediata segundo características socioeconômicas e de utilização de serviços e ações de saúde bucal nas três ondas. Sobral, 2012.

\begin{tabular}{|c|c|c|c|c|}
\hline Variável & $\mathbf{N}$ & Média (DP) & RR (IC) & $\mathbf{P}$ \\
\hline \multicolumn{5}{|l|}{ Variáveis da infância (2000) } \\
\hline \multicolumn{5}{|l|}{ Renda Familiar } \\
\hline Até $1 \mathrm{SM}^{*}$ & 132 & $1,01(1,88)$ & & \\
\hline Acima de $1 \mathrm{SM}$ & 350 & $1,42(2,07)$ & $1,41(0,99-2,00)$ & 0,03 \\
\hline \multicolumn{5}{|l|}{ Renda do chefe da família } \\
\hline Até $1 \mathrm{SM}$ & 228 & $0,95(1,67)$ & & \\
\hline Acima de $1 \mathrm{SM}$ & 241 & $1,64(2,30)$ & $1,73(1,29-2,30)$ & $<0,001$ \\
\hline \multicolumn{5}{|c|}{ Escolaridade chefe da família } \\
\hline Até 7 anos & 391 & $1,17(1,89)$ & & \\
\hline 8 anos ou mais & 89 & $1,94(2,51)$ & $1,66(1,22-2,27)$ & 0,007 \\
\hline \multicolumn{5}{|l|}{ Tipo de construção } \\
\hline Taipa & 84 & $0,83(1,61)$ & & \\
\hline Alvenaria & 397 & $1,41(2,09)$ & $1,69(1,09-2,62)$ & 0,006 \\
\hline \multicolumn{5}{|c|}{ Consultou dentista último ano } \\
\hline Não & 200 & $1,06(1,84)$ & & \\
\hline Sim & 280 & $1,69(2,26)$ & $1,37(1,18-1,52)$ & 0,001 \\
\hline \multicolumn{5}{|l|}{ Onde consultou dentista } \\
\hline Público & 167 & $1,57(2,20)$ & & \\
\hline Privado & 18 & $2,72(2,60)$ & $1,73(1,07-2,79)$ & 0,04 \\
\hline \multicolumn{5}{|c|}{ Variáveis da adolescência (2006) } \\
\hline \multicolumn{5}{|c|}{ Renda Familiar } \\
\hline Até $1 \mathrm{SM}$ & 252 & $1,08(1,90)$ & & \\
\hline Acima de $1 \mathrm{SM}$ & 230 & $1,56(2,14)$ & $1,45(1,10-1,92)$ & 0,009 \\
\hline \multicolumn{5}{|l|}{ Tipo de escola } \\
\hline Pública & 431 & $1,18(1,92)$ & & \\
\hline Privada & 46 & $2,43(2,17)$ & $2,06(1,46-2,91)$ & 0,003 \\
\hline \multicolumn{5}{|l|}{ Aglomerado domiciliar } \\
\hline Acima de 1 & 233 & $1,08(1,80)$ & & \\
\hline Até 1 & 249 & $1,52(2,21)$ & $1,40(1,06-1,86)$ & 0,01 \\
\hline \multicolumn{5}{|c|}{ Consulta dentista no último ano } \\
\hline Não & 254 & $0,93(1,75)$ & & \\
\hline Sim & 227 & $1,74(2,23)$ & $1,47(1,29-1,60)$ & $<0,001$ \\
\hline Onde consultou dentista & 381 & $1,28(1,98)$ & & \\
\hline Público & 52 & $2,50(2,47)$ & $1,96(1,44-2,67)$ & 0,001 \\
\hline \multicolumn{5}{|l|}{ Privado } \\
\hline \multicolumn{5}{|l|}{ Variáveis da juventude (2012) } \\
\hline Renda familiar & 159 & $0,89(1,59)$ & & \\
\hline Até $1 \mathrm{SM}$ & 320 & $1,52(2,20)$ & $1,72(1,25-2,36)$ & $<0,001$ \\
\hline \multicolumn{5}{|l|}{ Acima de 1 SM } \\
\hline \multicolumn{5}{|l|}{ Tipo de escola } \\
\hline Pública & 210 & $1,28(2,04)$ & & \\
\hline Privada & 47 & $2,11(2,70)$ & $1,65(1,08-2,52)$ & 0,05 \\
\hline \multicolumn{5}{|l|}{ Aglomeração domiciliar } \\
\hline Acima de 1 & 164 & $0,94(1,67)$ & & \\
\hline Até 1 & 318 & $1,50(2,18)$ & $1,59(1,16-2,18)$ & 0,002 \\
\hline \multicolumn{5}{|c|}{ Consulta dentista no último ano } \\
\hline Não & 258 & $0,91(1,85)$ & & \\
\hline Sim & 224 & $1,77(2,13)$ & $1,49(1,31-1,62)$ & $<0,001$ \\
\hline \multicolumn{5}{|l|}{ Onde consultou dentista } \\
\hline Público & 274 & $0,97(1,58)$ & & \\
\hline Privado & 172 & $2,09(2,54)$ & $2,15(1,65-2,80)$ & $<0,001$ \\
\hline Grupo de adolescente & 267 & $1,08(1,79)$ & & \\
\hline Não & 215 & $1,59(2,26)$ & $1,47(1,11-1,93)$ & 0,008 \\
\hline Sim & & & & \\
\hline
\end{tabular}

* SM: salário mínimo 
Tabela 2. Modelo ajustado dos fatores determinantes da assistência odontológica imediata. Sobral, 2012.

\begin{tabular}{lcccc}
\hline \multicolumn{1}{c}{ Variável } & RR (IC) & p & $\begin{array}{c}\text { RR } \\
\text { ajustado (IC) }\end{array}$ & $\begin{array}{c}\text { P } \\
\text { Ajustado }\end{array}$ \\
\hline $\begin{array}{l}\text { Modelo 1: Variáveis da infância (2000)* } \\
\text { Renda do chefe da família acima 1 SM }\end{array}$ & $1,73(1,29-2,30)$ & $<0,001$ & $1,33(1,08-1,51)$ & 0,01 \\
$\quad \begin{array}{l}\text { Consultou dentista no último ano } \\
\text { Modelo 2: Variáveis da adolescência (2006)* }\end{array}$ & $1,47(1,29-1,60)$ & $<0,001$ & $1,31(1,09-1,48)$ & 0,008 \\
$\quad$ Estudar em escola privada & $2,06(1,46-2,91)$ & 0,003 & $1,33(1,01-1,54)$ & 0,04 \\
$\quad$ Consultou dentista no último ano & $1,47(1,29-1,60)$ & $<0,001$ & $1,43(1,24-1,58)$ & $<0,001$ \\
Modelo 3: Variáveis da juventude (2012) & & & & 0,007 \\
$\quad$ Renda familiar acima de 1 SM & $1,72(1,25-2,36)$ & $<0,001$ & $1,35(1,11-1,52)$ & 0,02 \\
$\quad \begin{array}{l}\text { Aglomeração domiciliar até 1 } \\
\text { Consulta dentista no último ano }\end{array}$ & $1,59(1,16-2,18)$ & 0,002 & $1,29(1,04-1,48)$ & $<0,001$ \\
Participa de grupo de adolescentes & $1,49(1,31-1,62)$ & $<0,001$ & $1,43(1,24-1,57)$ & 0,02 \\
\hline
\end{tabular}

*Ajustado pela renda familiar atual.

ração familiar) apresentaram maior risco de não ter assistência odontológica. Travassos e Castro ${ }^{14}$ afirmam que iniquidades no acesso e utilização de serviços de saúde expressam oportunidades diferenciadas em função da posição social do indivíduo.

Apesar de não ter utilizado como desfecho o uso de serviços, e sim a assistência odontológica, o presente estudo permitiu, mesmo que de forma indireta, tal análise, pois, para avaliar a assistência odontológica recebida ao longo do curso de vida, pressupõe-se utilizar o serviço. Dessa forma, assim como outros estudos, observou-se a influência de condições socioeconômicas, tais como renda e escolaridade dos pais, sob a utilização dos serviços odontológicos ${ }^{11,15-21}$.

No Brasil, houve uma redução das desigualdades no acesso e aumento na utilização de serviços odontológicos entre os anos de 2003 e 2008, entretanto as iniquidades entre os grupos sociais ainda existem ${ }^{6}$. Pois, ainda se observa que áreas com maior necessidade de saúde, inclusive odontológica, e maior privação social são as que apresentam maior dificuldade de acesso aos serviços de saúde ${ }^{7,22}$.

O modelo conceitual do curso de vida (life course), proposto por Kuh e Ben-Schlomo ${ }^{8}$, considera que a saúde-doença é um processo dinâmico e que a exposição aos determinantes da saúde em diferentes estágios da vida podem melhorar ou piorar a saúde dos indivíduos. As desvantagens sociais na infância, bem como sua acumulação de forma incremental ao longo da vida, apresentam impacto na saúde bucal ${ }^{9,23}$. A influência do ciclo vital foi comprovada neste estudo visto que as condições socioeconômicas analisadas em diferentes estágios da vida (infância, adolescência e juventude) apresentaram relação com a trajetória da assistência odontológica. Para a assistência odontológica imediata, a infância e a adolescência se configuraram como períodos críticos, enquanto que para a ausência de assistência, a adolescência é um período crítico, independente do jovem estar desempregado no momento atual. Para ambos os desfechos, a situação atual apresentou-se como fator de risco. Essa análise longitudinal, torna este estudo relevante, visto que a maioria das pesquisas que avaliam a utilização dos serviços são de caráter transversal ${ }^{6,7,11,15-21}$.

Além dos fatores socioeconômicos, a assistência odontológica apresentou relação com as variáveis do serviço, o qual constatou que utilizar o serviço privado implicou em maior média de dentes com assistência odontológica imediata. Enquanto aqueles que não receberam informação de saúde bucal na adolescência e que consultaram o dentista há mais de um ano na juventude apresentaram maior número de dentes sem assistência odontológica.

Outro resultado relevante deste estudo foi a relação do capital social com a assistência odontológica. Verificou-se que os jovens que nunca participaram de grupos de adolescentes, independente da sua natureza, sejam grupos de esporte, dança, manifestações culturais ou política dentro da escola ou em associações na comunidade, ou grupos de caráter religioso em igrejas, 
Tabela 3. Média de dentes sem assistência odontológica segundo características socioeconômicas e de utilização de serviços e ações de saúde bucal nas três ondas. Sobral, 2012.

\begin{tabular}{|c|c|c|c|c|}
\hline Variável & $\mathbf{N}$ & Média (DP) & RR (IC) & $\mathbf{P}$ \\
\hline \multicolumn{5}{|l|}{ Variáveis da infância (2000) } \\
\hline \multicolumn{5}{|l|}{ Renda Familiar } \\
\hline Acima de $1 \mathrm{SM}^{*}$ & 350 & $0,38(0,91)$ & & \\
\hline Até $1 \mathrm{SM}$ & 132 & $0,67(1,28)$ & $1,79(1,19-2,69)$ & 0,01 \\
\hline \multicolumn{5}{|l|}{ Renda do chefe da família } \\
\hline Acima de 1SM & 241 & $0,34(0,70)$ & & \\
\hline Até 1 SM & 228 & $0,58(1,28)$ & $1,69(1,15-2,49)$ & 0,01 \\
\hline \multicolumn{5}{|l|}{ Desnutrição infantil } \\
\hline Não & 343 & $0,36(0,92)$ & & \\
\hline Sim & 128 & $0,72(1,25)$ & $1,99(1,33-2,98)$ & 0,004 \\
\hline \multicolumn{5}{|c|}{ Variáveis da adolescência (2006) } \\
\hline \multicolumn{5}{|c|}{ Aglomeração domiciliar } \\
\hline Até 1 & 249 & $0,35(0,81)$ & & \\
\hline Acima de 1 & 233 & $0,57(1,21)$ & $1,62(1,09-2,39)$ & 0,02 \\
\hline \multicolumn{5}{|l|}{ Tipo de escola } \\
\hline Privado & 46 & $0,09(0,28)$ & & \\
\hline Público & 431 & $0,50(1,07)$ & $5,79(2,22-15,09)$ & $<0,001$ \\
\hline \multicolumn{5}{|l|}{ Onde consultou dentista } \\
\hline Privado & 52 & $0,15(0,46)$ & & \\
\hline Público & 381 & $0,47(1,01)$ & $3,07(1,34-7,06)$ & $<0,001$ \\
\hline \multicolumn{5}{|l|}{ Recebeu informação de SB } \\
\hline Sim & 343 & $0,38(0,85)$ & & \\
\hline Não & 135 & $0,64(1,31)$ & $1,69(1,11-2,58)$ & 0,03 \\
\hline \multicolumn{5}{|l|}{ Variáveis da juventude (2012) } \\
\hline Desempregado & 370 & $0,36(0,95)$ & & \\
\hline Não & 112 & $0,78(1,21)$ & $2,15(1,45-3,18)$ & 0,001 \\
\hline \multicolumn{5}{|l|}{ Sim } \\
\hline \multicolumn{5}{|l|}{ Aglomeração domiciliar } \\
\hline Até 1 & 318 & $0,35(0,86)$ & & \\
\hline Acima de 1 & 164 & $0,67(1,27)$ & $1,92(1,29-2,86)$ & 0,004 \\
\hline \multicolumn{5}{|c|}{ Consultou dentista último ano } \\
\hline Sim & 224 & $0,24(0,52)$ & & \\
\hline Não & 258 & $0,65(1,29)$ & $2,75(1,88-4,01)$ & $<0,001$ \\
\hline \multicolumn{5}{|l|}{ Onde consultou dentista } \\
\hline Privado & 172 & $0,27(0,75)$ & & \\
\hline Público & 274 & $0,58(1,18)$ & $2,11(1,31-3,41)$ & 0,001 \\
\hline \multicolumn{5}{|l|}{ Grupo de Adolescente } \\
\hline Sim & 215 & $0,30(0,64)$ & & \\
\hline Não & 267 & $0,58(1,24)$ & $1,93(1,32-2,83)$ & 0,001 \\
\hline
\end{tabular}

* SM: salário mínimo.

ou mesmo nos Centros de Referência Assistência Social (CRAS), bem como aqueles vinculados à unidade básica de saúde, apresentaram maior média de dentes sem assistência odontológica, independente da condição socioeconômica atual.

Capital social significa um conjunto de recursos inerente às relações sociais e familiares, bem como à organização social da comunidade, úteis para o desenvolvimento cognitivo ou social do indivíduo. Sua teoria postula que o nível de confiança interpessoal e comunitária, normas de reciprocidade, relações de solidariedade e nível de participação comunitária, como em grupos, constituem um tipo de recurso que pode facilitar e trazer benefícios, inclusive para a saúde ${ }^{24-28}$.

Tem-se observado uma menor autopercepção da saúde entre os indivíduos que não participam de grupos ou que não possuem relações de confiança nas suas redes de apoio, como escola e vizinhança ${ }^{26,29}$. Além disso, Pattussi et al. ${ }^{30}$ veri- 
Tabela 4. Modelo ajustado dos fatores determinantes da história de dentes sem assistência odontológica segundo a onda analisada. Sobral, 2012.

\begin{tabular}{lrrrr}
\hline \multicolumn{1}{c}{ Variável } & RR (IC) & P & RR ajustado (IC) & p ajustado \\
\hline $\begin{array}{l}\text { Modelo 1: variáveis da infância }(2000)^{*} \\
\quad \text { Desnutrição infantil }\end{array}$ & $1,99(1,33-2,98)$ & 0,004 & $1,76(1,17-2,66)$ & 0,007 \\
$\quad$ Modelo 1: Variáveis da adolescência (2006)* & & & & \\
$\quad$ Estudar em escola pública & $5,79(2,22-15,09)$ & $<0,001$ & $4,08(1,59-10,44)$ & 0,003 \\
Não recebeu informação saúde bucal & $1,69(1,11-2,58)$ & 0,03 & $1,71(1,14-2,58)$ & 0,01 \\
Modelo 2: Variáveis da juventude (2012) & & & & 0,002 \\
$\quad$ Estar desempregado & $2,15(1,45-3,18)$ & 0,001 & $1,87(1,26-2,77)$ & 0,01 \\
$\quad$ Aglomeração domiciliar acima de 1 & $1,92(1,29-2,86)$ & 0,004 & $1,61(1,09-2,39)$ & $<0,001$ \\
$\quad$ Consultou dentista há mais de 1 ano & $2,75(1,88-4,01)$ & $<0,001$ & $2,47(1,71-3,58)$ & 0,01 \\
\hline Não participa(ou) de grupo de adolescente & $1,93(1,32-2,83)$ & 0,001 & $1,58(1,09-2,29)$ & \\
\hline
\end{tabular}

* Ajustado pelo desemprego.

ficaram que quanto maior o grau de empoderamento, medido como uma dimensão do capital social, entre jovens, menor é a prevalência de cárie. Já Bezerra e Goes ${ }^{31}$ não encontraram relação do capital social em adolescentes e a utilização de serviços odontológicos.

A participação em grupos tem sido utilizada como indicador para medir capital social, acredita-se que ao fazer parte destes, a possibilidade de confiança entre os participantes e o empoderamento aumentam, além de favorecer o desenvolvimento de comportamentos saudáveis à saúde, estimular a autoestima, melhorar a autopercepção da saúde e o acesso ao serviço ${ }^{28}$. O apoio e o acesso à informação que os jovens inseridos em determinados grupos recebem podem estimular o autocuidado em saúde bucal, bem como a procura aos serviços odontológicos. Além disso, é comum o dentista inserido na Estratégia de Saúde da Família do município de Sobral organizar sua demanda clínica a partir da triagem de grupos existentes na comunidade, bem como desenvolver atividades de educação em saúde nele, corroborando com o outro resultado encontrado, de que não receber informação em saúde bucal durante a adolescência é fator de risco para a falta de assistência odontológica.

A limitação para esse resultado foi a utilização de apenas de um indicador para medir capital social, visto que trata-se de um conceito amplo de interação comunitária e interpessoal, bem como não foi possível aprofundar como foi a inserção do indivíduo dentro deste grupo. Porém, este estudo sinalizou que a não participação de adolescentes em grupos apresentou-se como fator de risco para a falta de assistência odontológica.
A desnutrição infantil já tem sido associada com a maior prevalência de cárie dentária, pois a deficiência nutricional quando ocorrida nos primeiros anos de vida pode ocasionar efeito deletério no desenvolvimento do esmalte dentário ${ }^{32}$. Mas, a desnutrição infantil enquanto fator de risco para a falta de assistência odontológica, além do risco biológico, deve ser considerada como um proxy de situação socioeconômica.

Este estudo revelou a presença de iniquidades na assistência odontológica, mesmo dentro de um sistema universal, visto que os jovens com melhores condições socioeconômicas e que utilizaram o serviço privado ao longo da vida apresentaram maior possibilidade de receber assistência odontológica imediata. Há sistemas de saúde que potencializam as desigualdades existentes na organização social, enquanto outros, aqueles de caráter universal e equânime, procuram compensar, pelo menos em parte, os resultados danosos da organização social sobre os grupos socialmente mais vulneráveis ${ }^{5}$. Entretanto, os municípios que apresentam um modelo de saúde bucal mais bem estruturado e com menor iniquidade, são aqueles que também apresentam melhores condições socioeconômicas e políticas públicas mais bem definidas (educação, emprego, renda, moradia) $)^{4,33}$.

Vale salientar que o município de Sobral reorientou seu modelo de saúde bucal, com a inserção do dentista na Estratégia de Saúde da Família, antes da implantação da política nacional de saúde bucal - Brasil Sorridente ${ }^{34}$. Apesar de somente uma pequena parcela dos jovens nunca ter tido acesso ao serviço odontológico ou ter tido acesso negado pelo menos uma vez na vida, esse 
resultado é relevante visto a cobertura de equipes de saúde da família e de saúde bucal no momento desse estudo ser de quase $100 \%$ no município de Sobral. Portanto, há a necessidade dos serviços de saúde bucal avançarem no que se refere à equidade da assistência. Dessa forma, sugere-se que os gestores reorientem o modelo de atenção de forma a reduzir as iniquidades na assistência odontológica ao longo da vida, com vistas a um modelo real de vigilância à saúde, com garantia da equidade e integralidade da atenção. Ressalta-se, ainda, a necessidade de desenvolver ações educativas voltadas para a promoção da saúde e empoderamento do adolescente sobre sua saúde bucal. Além disso, é fundamental o desenvolvimento de políticas sociais e econômicas mais justas, capazes de reduzir as desigualdades sociais, com garantia da inserção do jovem no mercado de trabalho e o fortalecimento de redes e grupos de apoio social na comunidade.

\section{Colaboradores}

A participação dos autores se deu da seguinte forma: AKM Teixeira participou de todas as etapas do manuscrito, AG Roncalli e LRA Noro contribuíram no delineamento da pesquisa, análise e interpretação dos dados e revisão crítica do manuscrito. 


\section{Referências}

1. Brasil. Ministério da Saúde (MS). Diretrizes da Política Nacional de Saúde Bucal. Brasília: MS; 2004.

2. Brasil. Ministério da Saúde (MS). SB Brasil 2010: Pesquisa Nacional de Saúde Bucal: Resultados principais. Brasília: MS; 2011.

3. Martelli PJL, Cabral APS, Pimentel FC, Macedo CLSV, Monteiro IS, Silva SF. Analysis of the oral health care model in cities of the state of Pernambuco. Cien Saude Colet 2008; 13(5):1669-1674.

4. Souza TMS, Roncalli AG. Oral health in the Brazilian Family Health Program: a health care model evaluation. Cad Saude Publica 2007; 23(11):2727-2739.

5. Barata RB. Como e por que as desigualdades sociais fazem mal à saúde. Rio de Janeiro: ed. Fiocruz; 2009.

6. Peres KG, Peres MA, Boing, AF, Bertoldi AD, Bastos JL, Barros AJD. Redução das desigualdades sociais na utilização de serviços odontológicos no Brasil entre1998 e 2008. Rev Saude Publica 2012; 46(2):250-258.

7. Peres MA, Iser BPM, Boing AF, Yokota RTC, Malta DC, Peres KG. Desigualdades no acesso e na utilização de serviços odontológicos no Brasil: análise do VIGITEL 2009. Cad Saude Publica. 2012; 28(Supl.):S90-S100.

8. Kuh D, Ben-Shlomo Y. A life course apporach to chronic disease epidemiology. $2^{\text {nd }}$ ed. Oxford: Oxford University Press; 2004.

9. Nicolau B, Thomson WM, Steele JG, Allinson PJ. Lifecourse epidemiology: concepts and theoretical models with particular reference to oral chronic conditions. Community Dent Oral Epidemiol 2007; 35(4):241-249.

10. Noro LRA, Roncalli AG, Mendes Junior FIR, Lima KC. Children oral health status from Sobral-Ceará. Stoma 2008; 88:29-34.

11. Noro LRA, Roncalli AG, Mendes Junior FIR, Lima KG. Use of dental care by children and associated factors in Sobral, Ceará State, Brazil, Ceará, Brasil. Cad Saude Publica 2008; 4(7):1509-1516.

12. Noro LRA, Roncalli AG, Mendes Junior FIR, Lima KG. Dental caries incidence in adolescents in a city Northeast Brazil, 2006. Cad Saude Publica 2009; 25(4):783790.

13. Brasil. Ministério da saúde (MS). SB Brasil 2010 - Pesquisa Nacional de Saúde Bucal: Manual da equipe de campo. Brasília: MS; 2009.

14. Travassos C, Castro MSM. Determinantes e desigualdades no acesso e utilização de serviços de saúde. In: Giovanella L, Escorel S, Lobato LVC, Noronha JC, Carvalho AI, organizadores. Políticas e Sistema de Saúde no Brasil. 2a ed. Rio de janeiro, Editora: Fiocruz; 2012. p. 183-206.

15. ArdenghiI TM, Piovesan C, Antunes JLF. Inequalities in untreated dental caries prevalence in preschool children in Brazil. Rev Saude Publica 2013; 47(3):1-8.

16. Frias AC, Antunes JLF, Junqueira SR, Narvai PC. Individual and contextual determinants of the prevalence of untreated caries in Brazil. Rev Panam Salud Publica 2007; (22)4:279-285.

17. Mariño R, Khan A, Tham R, Khew CW, Stevenson C. Pattern and factors associated with utilization of dental services among older adults in rural Victoria. Aust Dent J 2014; 59(4):504-510.

18. Badri P, Saltaji H, Flores-Mir C, Amin M. Factors affecting children's adherence to regular dental attendance: A systematic review. J Am Dent Assoc 2014; 145(8):817-828.
19. Camargo MBJ, Dumith SC, Barros, AJD. Regular use of dental care services by adults: patterns of utilization and types of services. Cad Saude Publica 2009; 25(9):1894-1906.

20. Camargo MBJ, Barros, AJD, Frazão P, Matijasevich A, Santos IS, Peres MA, Peres KG. Predictors of dental visits for routine check-ups and for the resolution of problems among preschool children. Rev Saude Publica 2012; 46(1):87-97.

21. Ferreira CO, Antunes JLF, Andrade FB. Factors associated with the use of dental services by elderly Brazilians. Rev Saude Publica 2013; 47(3):90-97.

22. Junqueira SR, Frias AC, Zilbovicius C, Araújo ME. Saúde bucal e uso dos serviços odontológicos em função do Índice de Necessidades em Saúde: São Paulo, 2008. Cien Saude Colet 2012; 17(4):1015-1024.

23. Thomson WM. Social inequality in oral health. Community Dent Oral Epidemiol 2012; 40(2):28-32.

24. Kawachi I, Kennedy BP, Lochner K, Prothrow-stith D. Social capital, income inequality, and mortality. Am J Public Health 1997; 87(9):1491-1498.

25. Watt RG. Emerging theories into the social determinants of health: implications for oral health promotion. Community Dent Oral Epidemiol 2002; 30(4):241-247.

26. Furuta M, Ekuni D, Takao S, Suzuki E, Morita M, Kawachi I. Social capital and self-rated oral health among young people. Community Dent Oral Epidemiol 2012; 40(2):97-104.

27. Pearce N, Davey SG. Is social capital the key to inequalities in health? Am J Public Health 2003; 93(1):122-129.

28. Pattussi MP, Moysés SJ, Junges JR, Sheiham A. Social capital and the research agenda in epidemiology. Cad Saude Publica 2006; 22(8):1525-1546.

29. Caetano SC, Silva CM, Vettore VM. Gender differences in the association of perceived social support and social network with self-rated health status among older adults: a population-based study in Brazil. BMC Geriatr 2013; 15(13):122.

30. Pattussi MP, Hardy R, Sheiham A. The potential impact of neighborhood empowerment on dental caries among adolescents. Community Dent Oral Epidemiol 2006; 34(5):344-350.

31. Bezerra IA, Goes PSA. Association between social capital and oral health conditions and behavior. Cien Saude Colet 2014; 19(6):1943-1950.

32. Oliveira LB, Sheiham A, Bõnecker M. Exploring the association of dental caries with social factors and nutritional status in Brazilian preschool children. Eur J Oral Sci 2008; 116(1):37-43.

33. Celeste RK, Nadanovsky P. How much of the income inequality effect can be explained by public policy? Evidence from oral health in Brazil. Health Policy 2010; 97(2-3):250-258.

34. Teixeira AH, Bezerra MM, Pinto VPT. Oral Health in the Family Health Strategy: Finding Out the Performance of the Teams in Sobral Ceará. Sanare 2005. 6(1):9-18

Artigo apresentado em 01/06/2015

Aprovado em 15/12/2015

Versão final apresentada em 17/12/2015 